\title{
ISOLAMENTO DA CELULOSE DE BUCHA VEGETAL (LUFFA CYLINDRICA) VIA DIFERENTES TRATAMENTOS QUÍMICOS PARA GERAÇÃO DE MATERIAL DE REFORÇO
}

\author{
N. A. DIAS ${ }^{1}$, M. P. C. XAVIER ${ }^{1}$, N. G. MALAQUIAS ${ }^{1}$, E. P. SANTOS $^{2}$, M. L. C. P. da \\ SILVA $^{1}$ \\ ${ }^{1}$ Universidade de São Paulo, Departamento de Engenharia Química \\ ${ }^{2}$ Instituto Nacional de Pesquisas Espaciais, Laboratório Associado de Sensores e Materiais \\ E-mail para contato: esterzinha.psantos@gmail.com
}

\begin{abstract}
RESUMO - As fibras lignocelulósicas apresentam-se como grande atrativo na produção de novos materiais, principalmente como reforço em matrizes poliméricas, por serem renováveis, atóxicas e abundantes. Além disso, devido à presença de grupos hidroxila, são susceptíveis à modificações químicas acarretando uma melhora em características superficiais como adesão, molhabilidade, tensão superficial ou porosidade. O objetivo do trabalho é avaliar qual a melhor proposta de tratamento para a bucha vegetal com o propósito de gerar material híbrido a ser aplicado como reforço. Para tal, foram avaliadas quatro metodologias de ataque químico: ataque com $\mathrm{NaOH} ; \mathrm{CH}_{3} \mathrm{COOH}$; $\mathrm{NaOH} / \mathrm{CH}_{3} \mathrm{COOH}$ e $\mathrm{CH}_{3} \mathrm{COOH} / \mathrm{NaOH}$. A bucha tratada foi caracterizada por TGA/DTG e DRX. Observação física da mesma mostra que o melhor tratamento foi com $\mathrm{NaOH}$, onde o material tratado manteve-se resistente e estruturado. Os resultados de DRX indicam que o índice de cristalinidade se mantém estável nas diferentes metodologias.
\end{abstract}

\section{INTRODUÇÃO}

A procura por novos materiais tem se tornado cada vez mais interessante, no que se refere à utilização de fibras naturais (lignocelulósicas) principalmente como reforço em matrizes poliméricas. A elevada disponibilidade destas fibras juntamente com a necessidade do emprego de fontes renováveis favorecem a produção de materiais com alto valor agregado (SILVA et al. 2009).

Além da elevada disponibilidade e diversidade, as fibras naturais são renováveis, abundantes, atóxicas, de baixo custo, de baixa densidade e não abrasivas (ALBINANTE et al., 2013). Das diversas fibras utilizadas, a bucha vegetal (Luffa cylindrica) possui como grande vantagem a característica de manta natural ou estrutura vazada, dependendo do corte que lhe for aplicado. Este aspecto estruturado pode favorecer seus compósitos em determinadas propriedades mecânicas, pois os defeitos são defletidos na interface fibra/matriz (D'ALMEIDA et al., 2005). Entretanto, para uma maior adesão nesta interface e consequente melhora de propriedades, as modificações químicas apresentam-se como alternativa no favorecimento da interação entre os componentes devido à reação da fibra e do componente químico empregado, formando ligações covalentes, de hidrogênio ou ácido-base (SANCHEZ et al., 2010; ALBINANTE et al., 2013). 
Várias substâncias químicas vem sendo utilizadas no tratamento das fibras, tais como ácido acético, silano, ácido acrílico, isocianatos, permanganato de potássio, peróxidos, dentre outros (LI et al., 2007). O tratamento alcalino (mercerização) é o mais popular e barato processo para a modificação das fibras naturais, pois aumenta sua rugosidade superficial e melhora sua aderência mecânica, quesitos necessários quando as mesmas são empregadas como reforço em matrizes poliméricas (SANCHEZ et al., 2010; ALBINANTE et al., 2013). Contudo, com o intuito de minimizar as agressões ambientais dos diversos componentes tóxicos utilizados, outros tipos de tratamentos químicos estão sendo propostos, como por exemplo, a utilização de uma mistura ácida de $\mathrm{HNO}_{3} / \mathrm{CH}_{3} \mathrm{COOH}$ (BRENDEL et al., 2000; SILVA, 2013).

Neste contexto, este trabalho tem como objetivo avaliar qual a melhor proposta de tratamento para a bucha vegetal e verificar sua influência em suas características morfológicas, estruturais e térmicas.

\section{MATERIAIS E MÉTODOS}

\subsection{Materiais}

As buchas vegetais (Luffa cylindrica) foram previamente, limpas, secas e cortadas em rodelas de aproximadamente $1,5 \mathrm{~cm}$ de espessura. No ataque ácido utilizou-se uma mistura ácida de ácido acético $80 \% \mathrm{v} / \mathrm{v}$ e ácido nítrico $65 \% \mathrm{~m} / \mathrm{m}$. No ataque básico uma solução de hidróxido de sódio $0,1 \mathrm{~mol} / \mathrm{L}$.

\subsection{Tratamentos químicos}

Para verificar qual a melhor proposta de tratamento químico para as buchas vegetais foram avaliadas as seguintes metodologias, segundo a Tabela 1.

Tabela 1 - Tratamentos químicos empregados nas buchas vegetais

\begin{tabular}{|c|c|}
\hline MATERIAL & TRATAMENTO QUÍMICO \\
\hline Bucha básica & 1 ataque básico \\
\hline Bucha básica/ácida & 1 ataque básico +1 ataque ácido \\
\hline Bucha ácida & 1ataque ácido \\
\hline Bucha ácida/básica & 1 ataque ácido +1 ataque básico \\
\hline
\end{tabular}

Ataque ácido: para este tratamento empregou-se uma adaptação da metodologia proposta por Brendel et al. (2000). As buchas, previamente limpas e secas, foram colocadas em um béquer e adicionada uma mistura de ácidos acético/nítrico. O sistema foi mantido sob agitação a $120{ }^{\circ} \mathrm{C}$ por $20 \mathrm{~min}$ e, após aquecimento, filtrado a vácuo e lavado com água deionizada até $\mathrm{pH}$ neutro. Em seguida, as buchas foram secas em estufa a $50{ }^{\circ} \mathrm{C}$ até peso constante. 
Ataque básico: as buchas foram transferidas para um béquer e imersas em solução de $\mathrm{NaOH}$ 0,1 mol/L e mantidas sob aquecimento, por 20 minutos, após a solução atingir a ebulição. Em seguida, foram filtradas a vácuo e lavadas com água deionizada até pH neutro. As buchas foram secas em estufa a $50^{\circ} \mathrm{C}$ até peso constante.

\subsection{Caracterizações}

As buchas pura (sem tratamento) e tratadas quimicamente foram caracterizadas por termogravimetria e sua derivada (TGA/DTG), difração de raios X (DRX) e avaliação macroscópica.

A cristalinidade das buchas pura e tratadas foi determinada por DRX, em um difratômetro PANalytical, modelo Empyrean, com fonte de radiação $\mathrm{CuK} \alpha$, tensão de $40 \mathrm{kV}$ e corrente de $30 \mathrm{~mA}$, para valores de $2 \theta$ entre 10 e $90^{\circ}$.

O comportamento térmico de todos os materiais foi avaliado por TGA, em uma termobalança Shimadzu, modelo TGA-50, sob atmosfera de nitrogênio, com vazão de 50 $\mathrm{mL} / \mathrm{min}$ e taxa de aquecimento de $20^{\circ} \mathrm{C} / \mathrm{min}$, no intervalo da temperatura ambiente até $900^{\circ} \mathrm{C}$.

Realizou-se uma avaliação macroscópica das buchas tratadas quimicamente com o objetivo de verificar se sua estrutura foi mantida.

\section{RESULTADOS E DISCUSSÃO}

\subsection{Difração de raios $X$, DRX}

A Figura 1 apresenta os difratogramas para as buchas pura e tratadas quimicamente.

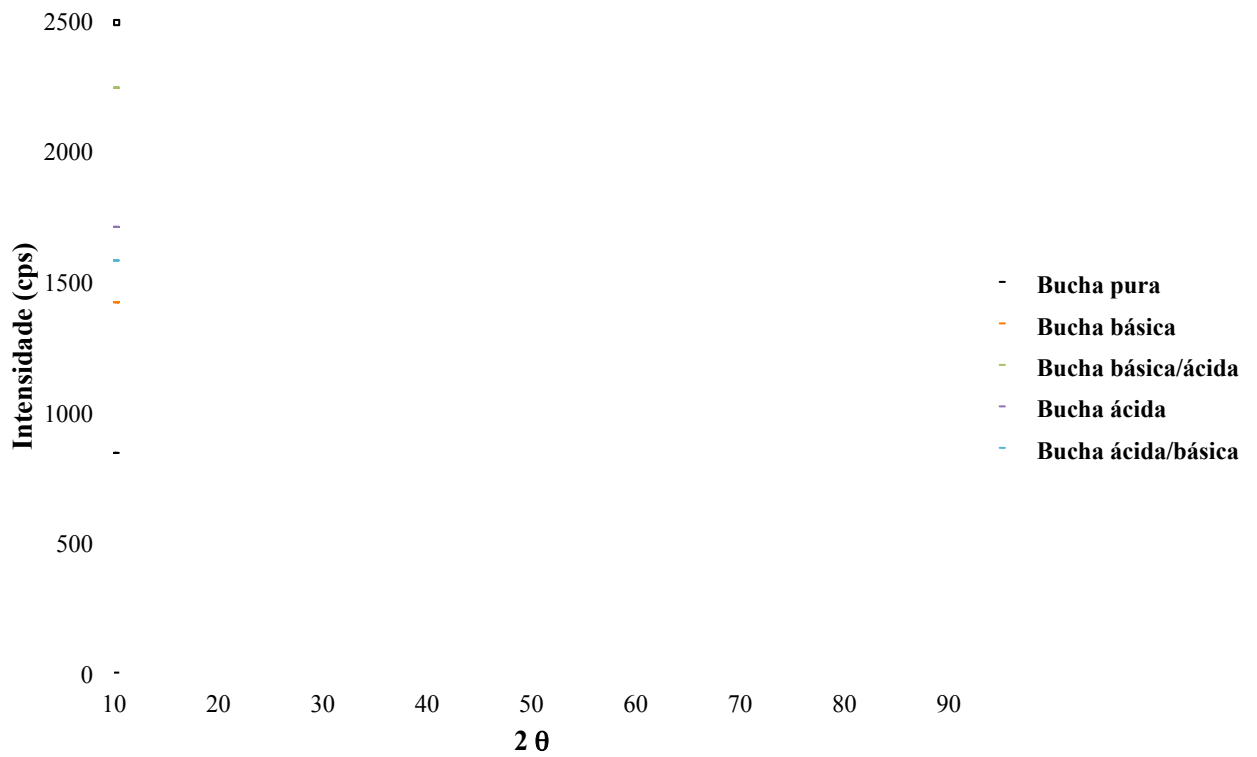

Figura 1 - Difratogramas de raios X para buchas pura e tratadas quimicamente. 
De acordo com a Figura 1, os difratogramas das buchas tratadas exibem três picos característicos de $2 \theta=15^{\circ}, 22^{\circ}$ e $35^{\circ}$, os quais correspondem aos planos cristalográficos (101), (002) e (040), respectivamente. Isto é, estes picos referem-se à celulose isolada após os tratamentos químicos empregados, os quais são intrínsecos das fibras lignocelulósicas (GUIMARÃES et al., 2010).

Baseado nos resultados obtidos nos difratogramas foi possível estimar o índice de cristalinidade $\left(I_{c}\right)$ da fração celulósica, definido pela Equação 1 (BUSCHLE-DILLER e ZERONIAN, 1992; GUIMARÃES et al., 2009):

$$
I_{c}=1-\frac{I_{M I N}}{I_{M A ́ X}} \times 100
$$

$\mathrm{I}_{\mathrm{MIN}}=$ intensidade mínimo do vale, referente ao material amorfo, na região de $2 \theta=18^{\circ}$;

$\mathrm{I}_{\mathrm{MÁX}}=$ intensidade máxima do pico de difração, referente ao material cristalino, na região de $2 \theta=22^{\circ}$.

A Tabela 2 exibe os valores dos $I_{c}$ para as buchas pura e tratadas quimicamente.

Tabela 2 - Valores dos índices de cristalinidade $\left(I_{c}\right)$

\begin{tabular}{|c|c|c|c|}
\hline Material & $\mathbf{I}_{(\mathbf{0 0 2})}$ & $\mathbf{I}_{\mathbf{a m}}$ & $\mathbf{I}_{\mathbf{c}}(\mathbf{\%})$ \\
\hline Bucha pura & 730,1 & 481,2 & 34,1 \\
\hline Bucha básica & 1228,7 & 651,5 & 47,0 \\
\hline Bucha básica/ácida & 1838,1 & 748,8 & 59,3 \\
\hline Bucha ácida & 1491,7 & 662,4 & 55,6 \\
\hline Bucha ácida/básica & 1300,6 & 671,1 & 48,4 \\
\hline
\end{tabular}

Segundo a Tabela 2, há o aumento significativo do $I_{c}$, comparado à bucha pura (sem tratamento) devido à eliminação das frações amorfas (lignina e hemicelulose), confirmando o isolamento da celulose (fração cristalina) de maneira eficaz em todos os tratamentos químicos (PEREIRA et al., 2014). Entretanto, os índices de cristalinidade apresentaram um valor maior para as buchas básica/ácida (59,3\%) e ácida (55,6\%), comparado aos índices referentes aos outros tratamentos químicos estudados. Este aumento pode estar relacionado ao tratamento químico empregado, seja ele de forma isolada ou finalizando o ataque químico (ataque ácido; básico/ácido). De acordo com Cao e Tan (2002), a mistura de ácidos promove uma maior degradação preferencialmente nas regiões amorfas, preservando a fração cristalina, o que contribui para o aumento na cristalinidade. Para as buchas básica e ácida/básica o comportamento foi oposto, comparado aos outros dois tratamentos, com uma redução dos índices. Esta redução também está relacionada ao tratamento empregado, pois o ataque alcalino aumenta a quantidade de celulose amorfa devido à fragilização da ligação hidrogênio 
na estrutura molecular da celulose, contribuindo assim para a diminuição no índice de cristalinidade (ALBINANTE et al., 2013). Devido à similaridade dos resultados, não foi possível definir o melhor tratamento químico. Esta definição deverá ser dada por meio de análises físicas.

\subsection{Termogravimetria, TGA/DTG}

A Tabela 3 apresenta os dados das curvas TGA/DTG para buchas pura e tratadas quimicamente, em atmosfera de nitrogênio.

Tabela 3 - Dados das curvas TGA/DTG para as buchas pura e tratadas quimicamente

\begin{tabular}{|c|c|c|c|c|}
\hline MATERIAL & $\begin{array}{c}\text { Intervalos } \\
\left({ }^{\circ} \mathrm{C}\right)\end{array}$ & $\begin{array}{c}\text { Perda de } \\
\text { massa }(\%)\end{array}$ & $\begin{array}{l}\text { Temp. pela } \\
\text { DTG }\left({ }^{\circ} \mathrm{C}\right)\end{array}$ & $\begin{array}{c}\text { Resíduo } \\
(\%)\end{array}$ \\
\hline BUCHA PURA & $\begin{array}{c}25-170 \\
170-525 \\
525-900\end{array}$ & $\begin{array}{c}7,33 \\
67,66 \\
17,48\end{array}$ & $\begin{array}{c}72 \\
377 \\
-\end{array}$ & 7,53 \\
\hline BUCHA BÁSICA & $\begin{array}{c}25-185 \\
185-440 \\
440-900\end{array}$ & $\begin{array}{c}6,70 \\
63,65 \\
24,61\end{array}$ & $\begin{array}{c}67 \\
355 \\
-\end{array}$ & 5,04 \\
\hline $\begin{array}{c}\text { BUCHA } \\
\text { BÁSICA/ÁCIDA }\end{array}$ & $\begin{array}{c}25-170 \\
170-460 \\
460-900\end{array}$ & $\begin{array}{c}6,05 \\
79,46 \\
10,78\end{array}$ & $\begin{array}{c}63 \\
360 \\
-\end{array}$ & 3,71 \\
\hline BUCHA ÁCIDA & $\begin{array}{c}25-180 \\
180-450 \\
450-900\end{array}$ & $\begin{array}{c}3,99 \\
74,85 \\
16,03\end{array}$ & $\begin{array}{c}73 \\
373 \\
-\end{array}$ & 5,13 \\
\hline $\begin{array}{c}\text { BUCHA } \\
\text { ÁCIDA/BÁSICA }\end{array}$ & $\begin{array}{c}25-190 \\
190-410 \\
410-900\end{array}$ & $\begin{array}{c}2,92 \\
76,35 \\
13,72\end{array}$ & $\begin{array}{c}64 \\
361 \\
598\end{array}$ & 7,01 \\
\hline
\end{tabular}

As curvas TGA/DTG para as buchas pura e tratada apresentam três eventos térmicos distintos: desidratação, decomposição e degradação, respectivamente. Segundo a Tabela 3, a bucha ácida apresenta a perda de massa $74,85 \%$ no evento de decomposição e a maior temperatura pela DTG $\left(373{ }^{\circ} \mathrm{C}\right)$, quando comparada às outras buchas. Essa temperatura é a mais próxima da bucha pura e o perfil da curva TGA (Figura 2) indica que esta bucha ácida é perdida continuamente ao longo do tempo até $900{ }^{\circ} \mathrm{C}$. 


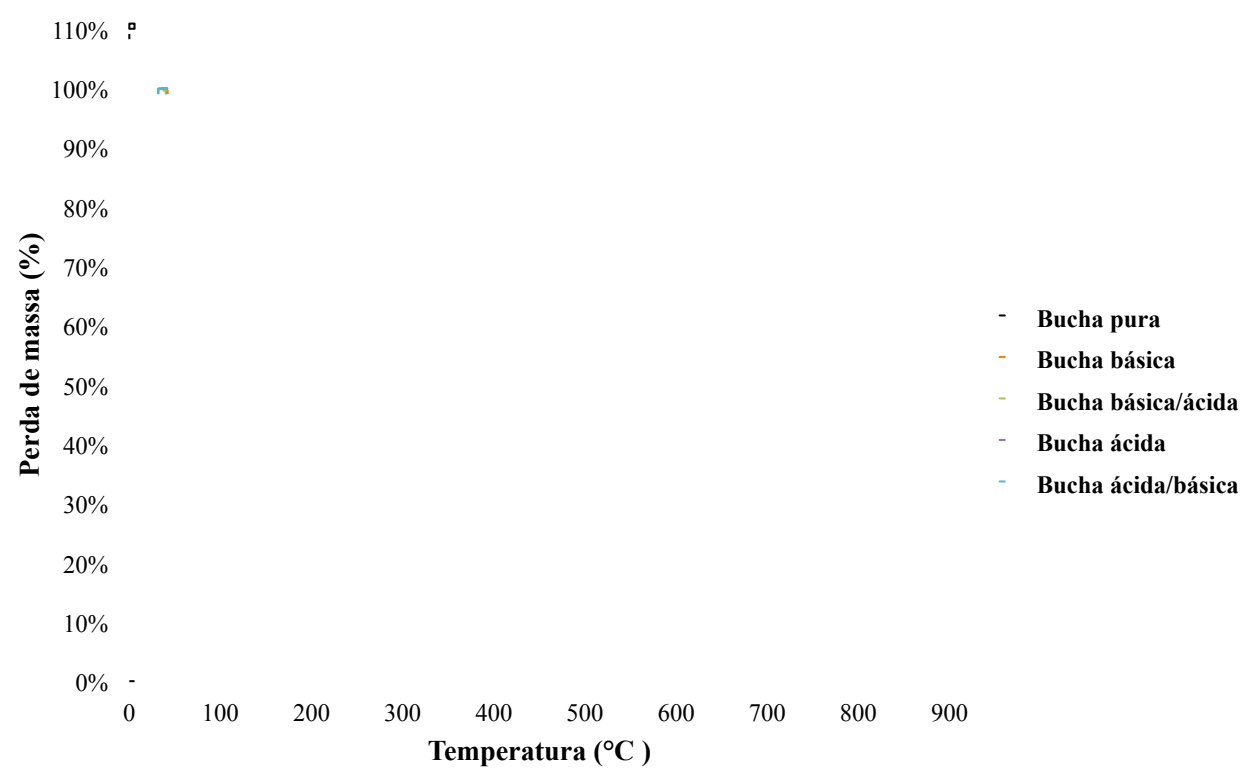

Figura 2 - Curvas TGA/DTG para as buchas pura e tratadas quimicamente.

\subsection{Avaliação macroscópica}

As buchas tratadas quimicamente foram submetidas a uma avaliação macroscópica e suas características encontram-se na Tabela 4.

\begin{tabular}{|c|c|}
\hline MATERIAL & CARACTERÍSTICA MORFOLÓGICA \\
\hline Bucha básica & Estrutura preservada e resistente \\
\hline Bucha básica/ácida & $\begin{array}{c}\text { Estrutura preservada, porém pouco resistente } \\
\text { e quebradiça }\end{array}$ \\
\hline Bucha ácida & $\begin{array}{c}\text { Estrutura preservada, porém extremamente } \\
\text { quebradiça }\end{array}$ \\
\hline Bucha ácida/básica & Ausência de estrutura \\
\hline
\end{tabular}

De com as características apresentadas na Tabela 4, pode-se definir a bucha básica como o melhor material obtido, pois sua estrutura vazada foi preservada e a mesma mantevese resistente. Apesar da bucha ácida exibir um índice de cristalinidade de 55,6 \% e uma temperatura de decomposição de $373{ }^{\circ} \mathrm{C}$ sua estrutura apresentou-se extremamente quebradiça, desqualificando sua escolha e, consequentemente, o ataque ácido de forma isolada. Portanto, a avaliação macroscópica foi crucial na determinação do tratamento químico devido à preservação estrutural da bucha, quesito interessante na geração compósitos poliméricos estruturados. 


\section{CONCLUSÃO}

A técnica de DRX não foi decisiva para a escolha do melhor tratamento químico, pois seus resultados apresentaram-se semelhantes.

As curvas TGA/DTG apresentaram-se como um indicativo para a avaliação do comportamento térmico.

A avaliação macroscópica foi determinante para a escolha do tratamento químico, pois somente na bucha básica (obtida a partir de um ataque básico) a estrutura foi mantida, com uma alta resistência.

\section{REFERÊNCIAS}

ALBINANTE, S. R. et al. Revisão dos tratamentos químicos da fibra natural para mistura com poliolefina. Quim. Nova, v. 36, n. 1, 114-122, 2013.

BRENDEL, O. et al. A rapid and simple method to isolate pure alpha-cellulose. Phytochem. Anal., v. 11, p. 7-10, 2000.

BUSCHLE-DILLER, G.; ZERONIAN, S. H. Enhancing the reactivity and strength of cotton fibers. J. Appl. Polym. Sci., v. 45, p. 967-979, 1992.

CAO, Y.; TAN, H. Effects of cellulase on the modification of cellulose. Carbohydr. Res., v. 337, p. 1291-1296, 2002.

D’ALMEIDA, A. L. F. S et al. Acetilação da fibra de bucha (Luffa cylindrica). Pol.: Ciência e Tecnol., v. 15, n. 1, p. 59-62, 2005.

GUIMARÃES, J. L. et al. Characterization of banana, sugarcane bagasse and sponge gourd fibres of Brazil. Ind. Crop. Prod., v. 30, p. 407-415, 2009.

GUIMARÃES, J. L. et al. Studies of the processing and characterization of corn starch and its composites with banana and sugarcane fibers from Brazil. Carbohydr. Polym., v. 80, p. 130$138,2010$.

LI, X. et al. Chemical treatments of natural fiber for use in natural fiber-reinforced composites: a review. J. Polym. Environ., v.15, p. 25-33, 2007.

MONTEIRO, S. N. et al. Thermogravimetric stability behavior of less common lignocellulosic fibers - a review. J. Mater. Res. Technol. v. 1, n. 3, p. 189-199, 2012.

PEREIRA, P. H. F. et al. Sugarcane bagasse cellulose fibres and their hydrous niobium phosphate composites: synthesis and characterization by XPS, XRD and SEM. Cellulose, v. 21, p. 641-652, 2014. 
SANCHEZ, E. M. S. et al. Compósito de resina de poliéster insaturado com bagaço de canade-açúcar: influência do tratamento das fibras nas propriedades. Polím., v. 20, n. 3, p. 194$200,2010$.

SILVA, R. et al. Aplicações de fibras lignocelulósicas na química de polímeros e em compósitos. Quím. Nova, v. 32, n. 3, p. 661-671, 2009.

SILVA, L. P. Preparação e caracterização de híbridos de celulose do bagaço de cana-deaçúcar e óxido de alumínio hidratado para aplicação em membranas. 169f. Dissertação de Mestrado, Universidade de São Paulo, São Paulo, 2013. 